\title{
GEOFLOW: simulation of convection in a spherical shell under central force field
}

\author{
P. Beltrame ${ }^{1, *}$, V. Travnikov ${ }^{1}$, M. Gellert ${ }^{1}$, and C. Egbers ${ }^{1}$ \\ ${ }^{1}$ Department of Aerodynamics and Fluid Mechanics, Brandenburg University of Technology, Siemens-Halske-Ring 14, \\ 03046 Cottbus, Germany \\ *now at: Max-Planck-Institut, Dresden, Germany
}

Received: 4 August 2005 - Revised: 26 April 2006 - Accepted: 26 April 2006 - Published: 15 August 2006

Part of Special Issue "Turbulent transport in geosciences"

\begin{abstract}
Time-dependent dynamical simulations related to convective motion in a spherical gap under a central force field due to the dielectrophoretic effect are discussed. This work is part of the preparation of the GEOFLOW-experiment which is planned to run in a microgravity environment. The goal of this experiment is the simulation of large-scale convective motion in a geophysical or astrophysical framework. This problem is new because of, on the one hand, the nature of the force field (dielectrophoretic effect) and, on another hand, the high degree of symmetries of the system, e.g. the top-bottom reflection. Thus, the validation of this simulation with well-known results is not possible. The questions concerning the influence of the dielectrophoretic force and the possibility to reproduce the theoretically expected motions in the astrophysical framework, are open. In the first part, we study the system in terrestrial conditions: the unidirectional Earth's force is superimposed on the central dielectrophoretic force field to compare with the laboratory experiments during the development of the equipment. In the second part, the GEOFLOW-experiment simulations in weightless conditions are compared with theoretical studies in the astrophysical framework's, in the first instance a fluid under a selfgravitating force field. We present complex time-dependent dynamics, where the dielectrophoretic force field causes significant differences in the flow compared to the case that does not involve this force field.
\end{abstract}

\section{Introduction}

The present paper shows results of investigations of the influence of a radial force field, produced by the dielectrophoretic effect (Pohl , 1978) in spherical Rayleigh-Bénard convection using a three-dimensional code and bifurcation analysis. It is

Correspondence to: P. Beltrame

(beltrame@mpipks-dresden.mpg.de) the preparatory work for an experimental set-up: the convective motion in a spherical gap under the influence of an artificial central force field. This experiment is planned to run on the ISS (International Space Station) under microgravity conditions. Experimental details can be found in Egbers et al. (2003). The experimental cell is formed by an outer glass sphere, which can be cooled, and an inner sphere, which can uniformly heated within (Fig. 1). The temperature difference is maintained constant with $T_{1}>T_{2}$. The central force field is generated by applying a high voltage $(\simeq 10 \mathrm{kV})$ between inner and outer sphere. Using a dielectric fluid (silicon oil), the resulting central dielectrophoretic force field is proportional to $1 / r^{5}$. Currently, three different viscosities of fluid silicone oils and three different inner radii $\left(R_{1}\right)$ are available for the experiment (Table 1), resulting in three values of the Prandtl number, $\operatorname{Pr}$, and also three aspect ratios, $\eta$, respectively. The "central" Rayleigh number $R a_{c}$ can vary over a large range by varying the voltage (Table 1 ).

The possible nondimensional parameters have similar values to the Earth's mantle ones, in particular the aspect ratio, the Rayleigh number and, in both cases (GEOFLOW and Earth's mantle), $P r \gg 1$. The aspect ratio of the Earth's outer core $\left(\eta_{e}=0.34\right)$ is close to the GEOFLOW's ratio too. But the Prandtl number $(0.1<P r<10)$ is smaller than for the experiment and the very large Rayleigh number $\left(R a_{e}>10^{26}\right)$ cannot be achieved in the experiment. However, the rapid decay of the dielectrophoretic force field $\left(1 / r^{5}\right.$ variations) can better represent the gravity field of the outer core $\left(1 / r^{2}\right.$ variations) than the linear variation of the Earth's mantle gravity field.

Although the GEOFLOW-experiment allows the system to rotate, we consider here the non-rotating case in contrast to the Earth's case where the Taylor number plays a relevant role. This limiting case is motivated, on one hand, by the rich dynamics expected and, on another hand, by the possibility to interpret the results in theoretical way using group theory for the spherical symmetry: the $O(3)$ group. 


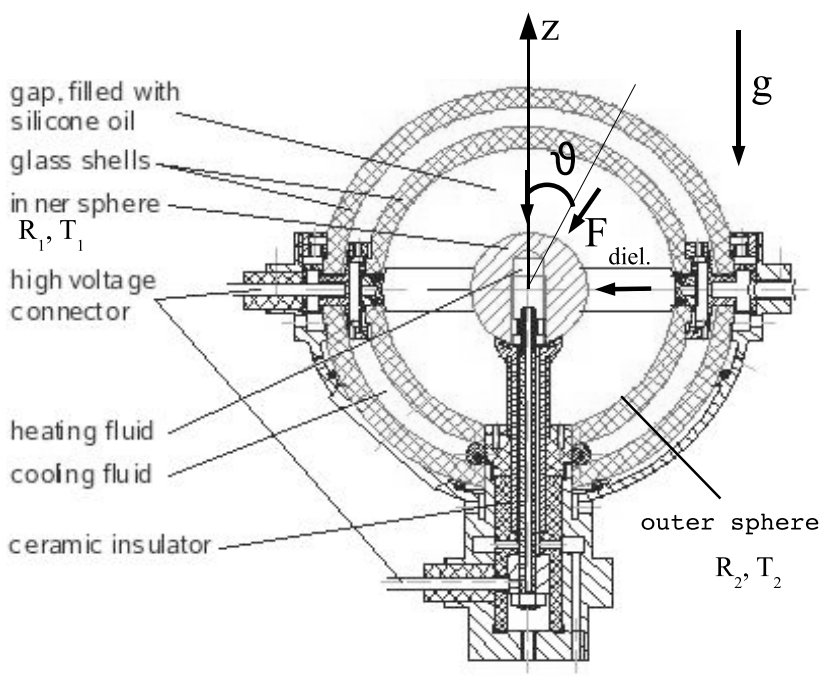

Fig. 1. Set-up of the GEOFLOW-experiment.

To obtain a perfect spherical symmetry, we have also neglected the thin axis supporting the inner sphere in the GEOFLOW-experiment as shown in Fig. 1. The thin axis will be taken into account in future works as a perturbation of the perfect case. Besides theoretical aspects, without this symmetry we could not use a pseudo-spectral method for the numerical computation, and the CPU time would then increase dramatically.

First results of numerical investigations, corresponding to convection in the rotating or non-rotating spherical gap under weightlessness conditions are published in Travnikov et al. (2003) and Travnikov (2004). These papers deal with the calculation of the basic flow, stability analysis and point out that the GEOFLOW-experiment can reproduce the different steady-states and rotating waves, which arise for a selfgravitating case $\left(1 / r^{2}\right.$ force field). Nevertheless, it is very difficult to validate these results with terrestrial experiments or well-known results, because the Earth's gravity field has a non-negligible influence, in particular for the non-rotating case where a lot of symmetry is broken. That is why we aim at simulating the system under two forces: the axial gravity force and the central dielectrophoretic force. Furthermore, we take the opportunity to point out the effect of the dielectrophoretic force compared with experimental/numerical work without this field (Futterer et al., 2004).

The second part of this paper considers the weightlessness case and it focuses on the comparison between the motion due to the dielectrophoretic field $\left(1 / r^{5}\right.$ radial dependence) and the central gravity field $\left(1 / r^{2}\right.$ radial dependence), which corresponds, for example, to the Earth's outer core. The $1 / r^{2}$ case has shown very rich dynamics, in particular the occurrence of motion reversals (Friedrich and Haken, 1986). This motion has astrophysical relevance because it can help to understand quasi-periodic phenomena such the Earth's mag- netic field reversal. Because Chossat and Guyard (1996) have pointed out that these reversal motions (or heteroclinic cycles) are due to the spherical symmetry, we expect such dynamics for the $1 / r^{5}$ field force too. The requirements on the GEOFLOW parameters which lead to possible heteroclinic cycles, are determined in Beltrame et al. (2003a) and Beltrame and Egbers (2004). The expected dynamics can be the same type as for the $1 / r^{2}$ case (Beltrame and Egbers, 2005) or can be new types of dynamics (Beltrame, 2006a ${ }^{1}$ ). In any cases, the dynamics are poorly known for both astrophysical $\left(1 / r^{2}\right)$ and dielectrophoretic $\left(1 / r^{5}\right)$ fields. Beyond these theoretical results, we will check the range of parameters for which these dynamics can be observed in GEOFLOW framework.

\section{Part I}

\section{Terrestrial conditions}

Four main non-dimensional numbers are necessary to describe the phenomenon: the radius ratio $\eta=\frac{R_{1}}{R_{2}}$, the Prandtl number $\operatorname{Pr}=\frac{\nu}{\kappa}$, the Rayleigh number $R a_{g}=\frac{\alpha g \Delta T R_{2}^{3}}{\nu \kappa}$ measuring the gravity force and the central Rayleigh number $R a_{c}=\frac{2 \epsilon_{0} \epsilon_{r} \gamma}{\rho_{0} v \kappa} V^{2} \Delta T$ measuring the dielectrophoretic force $\left(\epsilon_{0}\right.$ is the vacuum dielectric constant.) The notations are as follows: $R_{1}$ and $R_{2}$ are the radii of both spheres, $\alpha$ is the coefficient of volume expansion, $v$ the viscosity, $\kappa$ the thermal conductivity and $\rho_{0}$ the density. Furthermore, $\epsilon_{r}$ is the dielectric constant, $V$ the effective voltage and $\gamma$ the dielectric variability. This last constant is related to the dielectric constant linear dependence on the temperature: $\epsilon=\epsilon_{0} \epsilon_{r}\left(1-\gamma\left(T_{1}-T_{2}\right)\right)$. The investigation is performed for $\eta=0.5, \operatorname{Pr}=42.81$ (silicone oil M1). The temperature difference varies between $\Delta T=2$ and $\Delta T=8 \mathrm{~K}$. The flow structure then depends on the voltage $V$ for fixed $\Delta T\left(R a_{g}\right)$.

The goal of this part is to perform a numerical investigation of the influence of a fast oscillating electric field on the convective flow in the spherical gap in a terrestrial laboratory.

\section{Mathematical background}

\subsection{Basic equations}

We consider an incompressible, Newtonian fluid under the Boussinesq approximation. The force acting on the volume element of the dielectric medium, consists of three parts: Coulomb force $\mathbf{F}_{c}=\rho_{f r} \mathbf{E}$ ( $\rho_{f r}$ free charge density), dielectrophoretic force $\mathbf{F}_{d}=-\frac{1}{2} \mathbf{E}^{2} \nabla \epsilon$ and the gradient part

\footnotetext{
${ }^{1}$ Beltrame, P.: Intermittency between the Modes 3 and 4 near the onset of convection in a spherical shell under dielectrophoretic force, J. Adv. Space Res., in review, 2006a.
} 
Table 1. Mean physical and nondimensional parameters of GEOFLOW-experiment.

\begin{tabular}{|c|c|c|c|c|c|}
\hline Experiment parameters & & & & & \\
\hline Inner radius & $R_{1}$ & & $8.1-13.5$ & & $\mathrm{~mm}$ \\
\hline Outer radius & $R_{2}$ & & 27 & & $\mathrm{~mm}$ \\
\hline Temperature Difference & $\Delta T$ & & $2-6$ & & $\mathrm{~K}$ \\
\hline Voltage difference & V & & $0-12$ & & $\mathrm{kV}$ \\
\hline Fluid properties (at 25C) & & M1 & M2 & M3 & \\
\hline Density & $\rho_{0}$ & 0.90 & 0.92 & 0.94 & $\mathrm{~g} \mathrm{~cm}^{-3}$ \\
\hline Kinematic viscosity & $v$ & 3 & 5 & 10 & $\mathrm{~mm}^{2} \mathrm{~s}^{-1}$ \\
\hline Volume expansion coeff. & $\alpha$ & 1.11 & 1.08 & 1.03 & $10^{-3} \mathrm{~K}^{-1}$ \\
\hline Thermal diffusivity & $\kappa$ & & $77 \times 10^{-3}$ & & $\mathrm{~mm}^{2} \mathrm{~s}^{-1}$ \\
\hline Relative dielectric constant & $\epsilon_{r}$ & & 2.6 & & (at $800 \mathrm{~Hz}$ ) \\
\hline \multicolumn{6}{|l|}{ Adimensional parameters } \\
\hline Prandtl No. & $\mathrm{Pr}$ & 42(M1) & 107(M2) & 205(M3) & \\
\hline Aspect ratio & $\eta$ & 0.3 & 0.4 & 0.5 & \\
\hline Rayleigh No. (axisymmetric) & $\mathrm{Rag}$ & & $4 \times 10^{6}$ & & \\
\hline Rayleigh No. (central electric field) & $\mathrm{Ra}_{\mathrm{c}}$ & & $10^{3}-10^{7}$ & & \\
\hline
\end{tabular}

$\frac{1}{2} \nabla\left(\rho \frac{\partial \epsilon}{\partial \rho} \mathrm{E}^{2}\right)$. The last term can be combined with the pressure gradient. It can be shown that if the period of the Alternate Current (a.c.) electric field is much smaller than the relaxation time of free charge, the Coulomb force can be neglected. The detailed theory of the electrodynamics for this problem can be found in Yavorskaya et al. (1984).

The basic equations are the Navier-Stokes and Energy equations for an incompressible fluid, used here in a dimensionless version based on the following scaling: $r=R_{2} r^{*}$ for length, $T-T_{2}=\Delta T T^{*}$ for temperature, $\mathbf{U}=\frac{\kappa}{R_{2}} \mathbf{U}^{*}$ for velocity and $t=\frac{R_{2}^{2}}{\kappa} t^{*}$ for time.

The resulting system of non-dimensional equations (the superscript stars have been dropped)

$$
\begin{aligned}
\operatorname{Pr}^{-1}\left[\frac{\partial \mathbf{U}}{\partial t}+(\mathbf{U} . \nabla) \mathbf{U}\right]= & -\nabla \mathrm{P}_{\mathrm{eff}}+\operatorname{Ra}_{\mathrm{g}} T \mathbf{e}_{\mathrm{z}} \\
& +\frac{R a_{c}}{\beta^{2}} \frac{T}{r^{5}} \mathbf{e}_{\mathrm{r}}+\nabla^{2} \mathbf{U}
\end{aligned}
$$

$\frac{\partial T}{\partial t}+\mathbf{U} \cdot \nabla T=\nabla^{2} T$

$$
\nabla \cdot \mathbf{U}=0
$$

with $\beta=\frac{R_{2}-R_{1}}{R_{1}}$ together with no-slip boundary conditions for velocity components and constant temperatures on surfaces $\left(T_{1}>T_{2}\right)$ needs to be solved.

\subsection{Numerical method}

As mentioned in the introduction, the axis present in the experiment is neglected and then the domain is a perfect spherical shell. Thus the potentials of the poloidal-toroidal representations of the divergence-free velocity field (Eq. 3) together with the temperature are expanded in spherical harmonics:

$S(\mathrm{r}, \vartheta, \varphi)=\sum_{\ell=1}^{\mathrm{LU}} \sum_{\mathrm{m}=0}^{\mathrm{M}} a_{\ell \mathrm{m}}(\mathrm{r}) \mathrm{P}_{\ell}^{\mathrm{m}}(\cos \vartheta) e^{\mathrm{im} \varphi}$,

with $\mathrm{P}_{\ell}^{\mathrm{m}}$ the Legendre polynomials and $S$ an unknown scalar function. Each radial $a_{\ell \mathrm{m}}(\mathrm{r})$ function is discretized using the first kind Chebyshev polynomials $\mathrm{T}_{\mathrm{k}}$ :

$a_{\ell \mathrm{m}}(\mathrm{r})=\sum_{\mathrm{k}=1}^{\mathrm{KU}+2} b_{\mathrm{k} \ell \mathrm{m}} \mathrm{T}_{\mathrm{k}-1}(\mathrm{r})$.

The numbers KU, LU and M are cut off parameters.

The differentiation operators and the non-linear terms are computed using the well-known pseudo spectral method (Canuto et al., 1987). Its principle is to switch back and forth between spectral and real space: spectral space to do the derivatives and collocation points in real space to do the multiplications of the non-linear terms. The time-stepping is implemented using a second-order Runge-Kutta method, modified to treat diffusive terms implicitly. The detail of the numerical scheme is described in paper from Hollerbach (2000). 


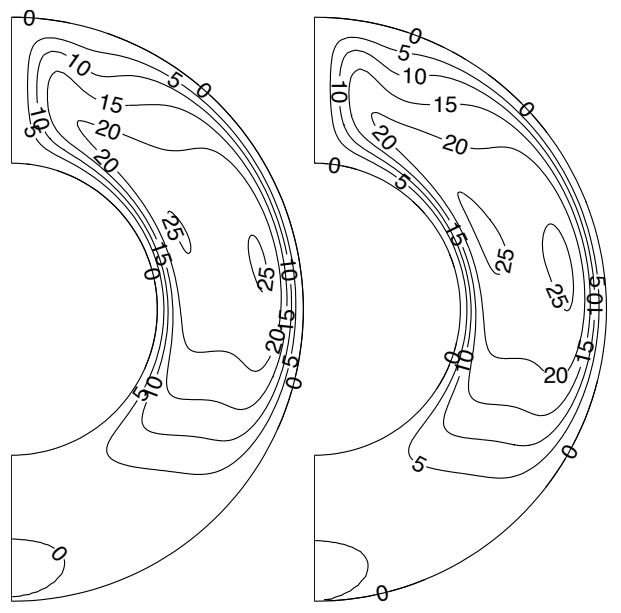

Fig. 2. Stationary flow: stream function for $\operatorname{Pr}=42.81, \eta=0.5$, $R a_{g}=4.0 \times 10^{6} \quad(\Delta T=4 \mathrm{~K}), \quad R a_{c}=0 \quad$ (left) and $R a_{c}=4.0 \times 10^{4}$ (right).

The simulations, in this part, are undertaken with $\mathrm{KU}=60$ (number of collocation points of the radial variable), $\mathrm{LU}=280$ (number of the spherical harmonics) and $\mathrm{M}=0$ (since the problem is axisymmetric.) The time step is around $5 \times 10^{-7}$. In this framework, we have needed several hours on a PowerPC (IBM F7040-671) CPU to obtain periodic solutions.

\section{Results}

In the case without an electric field a stationary flow of natural convection only can be observed (Fig. 2). This basic flow occurs because the gravity and the temperature gradient vectors are not parallel in comparison with the classical plane or spherical Rayleigh-Bénard convection without rotation. If the electric field is applied, the flow remains stationary as long as one is below a critical voltage for the fixed $\Delta T$.

For values larger than the critical voltage $V^{c}$ the flow becomes oscillatory. An example of the oscillating state can be seen in Fig. 3 which shows the behaviour of the temperature spectral coefficient and the kinetic energy. Figure 4 shows the time development of the stream function. This motion is characterized by the formation and decay of a vortex near the north pole of the spherical system.

These results can be compared with the experimental results from Amara et al. (2001) who have found that the flow "becomes unstable to toroidal or spiral rolls that form near the inner sphere and travel vertically upwards when $\Delta T$ and $\Delta V$ are sufficiently large." They have performed investigations for the silicon oil DC-200 with $P r=10.5$ (we have $P r=42.81$ ) and $\eta=0.37$ (we have $\eta=0.5$ ). The comparison with the experimental results shows the similar monotonically increasing of the frequency with $\Delta \mathrm{T}$, but in contrast to
Amara et al. (2001) the frequency depends slightly on the voltage $\mathrm{V}$ (Fig. 5).

Noticing that the critical $R a_{g}^{c}$ of the oscillations onset is a decreasing function of $R a_{c}$, we can thus conclude that $R a_{c}$ plays a relevant role for this instability but the frequency depends essentially on $\mathrm{Ra}_{\mathrm{g}}$.

Finally, let us mention another study simulating the superposition of vertical and central force fields using magnetic fluids as described in Früh (2005). The magnetic field produced approximatively a central $1 / r^{5}$-dependent field and in terrestrial conditions, the simulation resulted in regular oscillations where two kinds of vortices were in competition. The dynamics are more complex than our case and the geometry of vortices is not similar. These differences are expected since many differences exist between both experiments: among others no perfect central-symmetry of the field in Früh (2005) and different Rayleigh number values. However, their oscillating dynamics are reminiscent of our results, indicating that such an instability seems a robust phenomenon.

\section{Part II}

\section{Microgravity environment}

One important aspect of the GEOFLOW-experiment is the utilisation of a central force field, thus the spherical symmetry is respected. In the non-rotating case, the system is paradigmatic for the $O(3)$-equivariant bifurcation theory. The advantage to consider a perfect symmetry is that it gives a rich structure to the bifurcation problem. Furthermore, as Porter and Knobloch (2001) have pointed out, a lot of interesting dynamics are the consequence of symmetry imperfections and can be treated as a perturbation of the perfect symmetry using so-called perturbation theory. In our case, the imperfections can be due to the non-uniformity of the force field, or the presence of the thin axis in the experimental setup (Fig. 1). Also small rotation rates can be interpreted as a symmetry breaking from $O(3)$ to $S O(2) \oplus Z_{2}^{c}$ (P. Chossat, personal communication ${ }^{2}$ ).

However, there is a difference between GEOFLOW and the astrophysical framework: the variation of the simulated force field $\left(1 / r^{5}\right)$ is different from the encountered force field variations: $r$ dependence for high-density domain (e.g. Earth's mantle) and $1 / r^{2}$ dependence for low-density fluid surrounding a high density (e.g. Earth's outer core). Of course, this difference does not break the symmetry of the system, but the so-called "self-adjoint" degeneracy - responsible for the existence of reversal motions - no longer occurs (Chossat and Guyard, 1996). Indeed, this degeneracy comes from the anti-symmetry between the competing forces, i.e.

\footnotetext{
${ }^{2}$ Chossat, P.: Intermittency at onset of convection in a slowly rotating self-rotating spherical shell, private communication, 1999.
} 

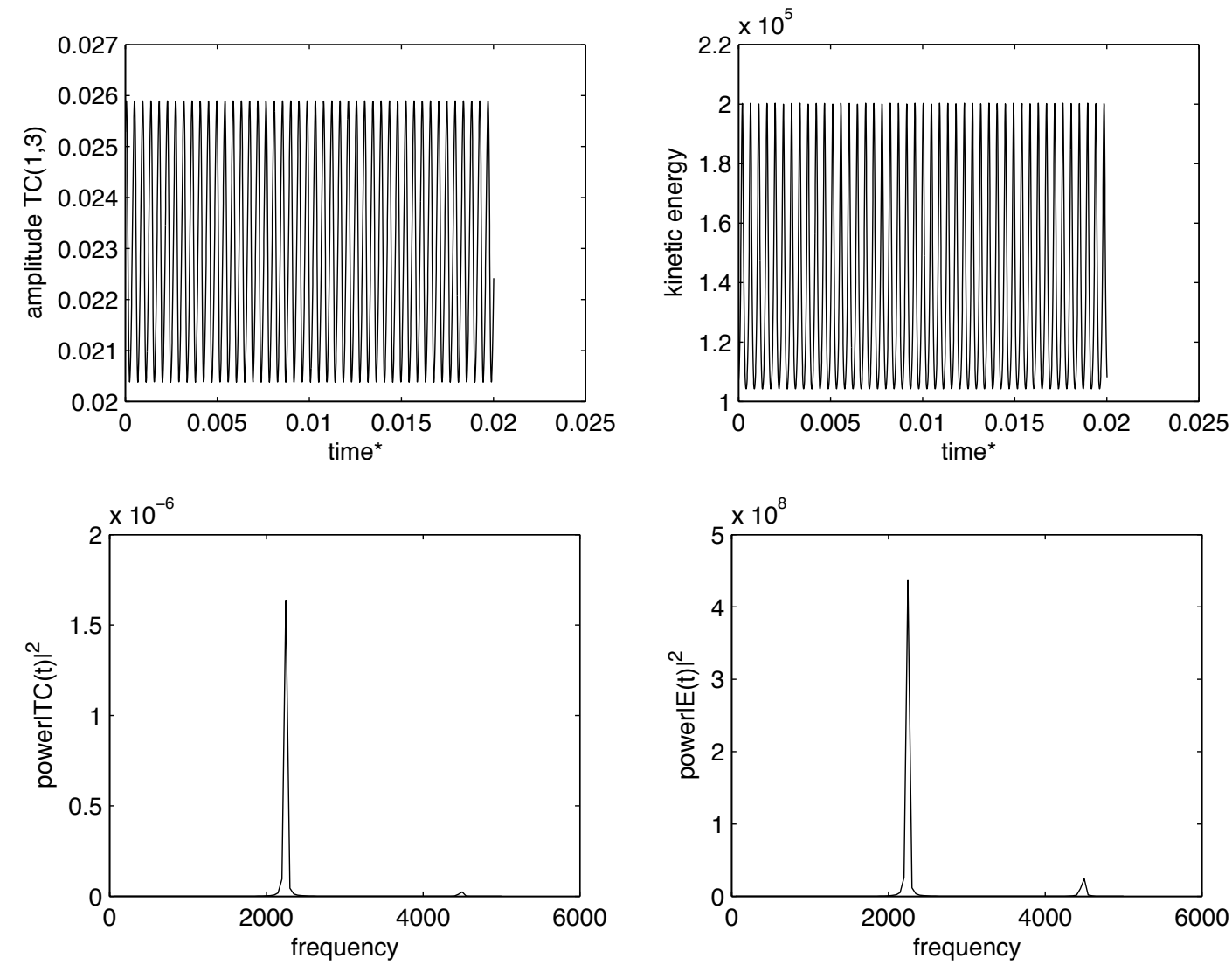

Fig. 3. Time dependent flow: spectral coefficients for the temperature (top left), kinetic energy (top right) and power spector for the spectral coefficient for the temperature (bottom left) and the kinetic energy (bottom right) $\operatorname{Pr}=42.81, \eta=0.5, R a_{g}=4.0 \times 10^{6}(\Delta T=4 \mathrm{~K})$, $R a_{c}=1.6 \times 10^{5}$.
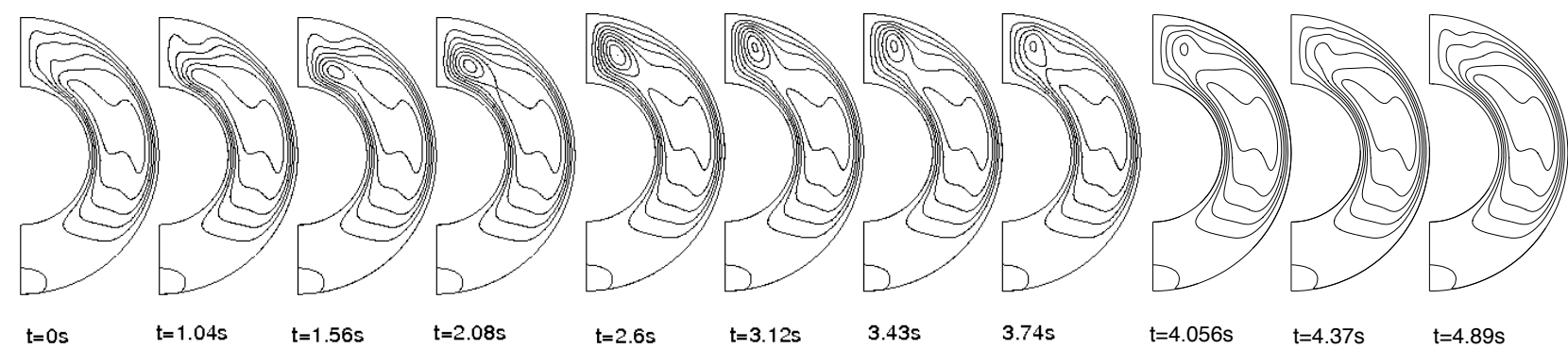

Fig. 4. Time dependent flow: Stream function for $\operatorname{Pr}=42.81, \eta=0$
consecutive contours is 5 .
he gravity force and the buoyancy gradient, both of which have $1 / r^{2}$ variations in that case. For that reason, we chose to compare the dynamics under dielectrophoretic force field with the dynamics under $1 / r^{2}$ field which is known to lead to the heteroclinic cycles. We aim at answering the questions:

- can the dielectrophoretic force field reproduce such complex dynamics?

- How do the dynamics differ between both cases?

Because of experimental requirements, we have restricted the study to aspect ratios $\eta>0.3$ but have covered a large range of Prandtl number.

\section{Bifurcation analysis method}

The mathematical modeling of the problem is the same as the system of Eqs. (1-2-3) by removing the $R a_{g}$ contribution in 

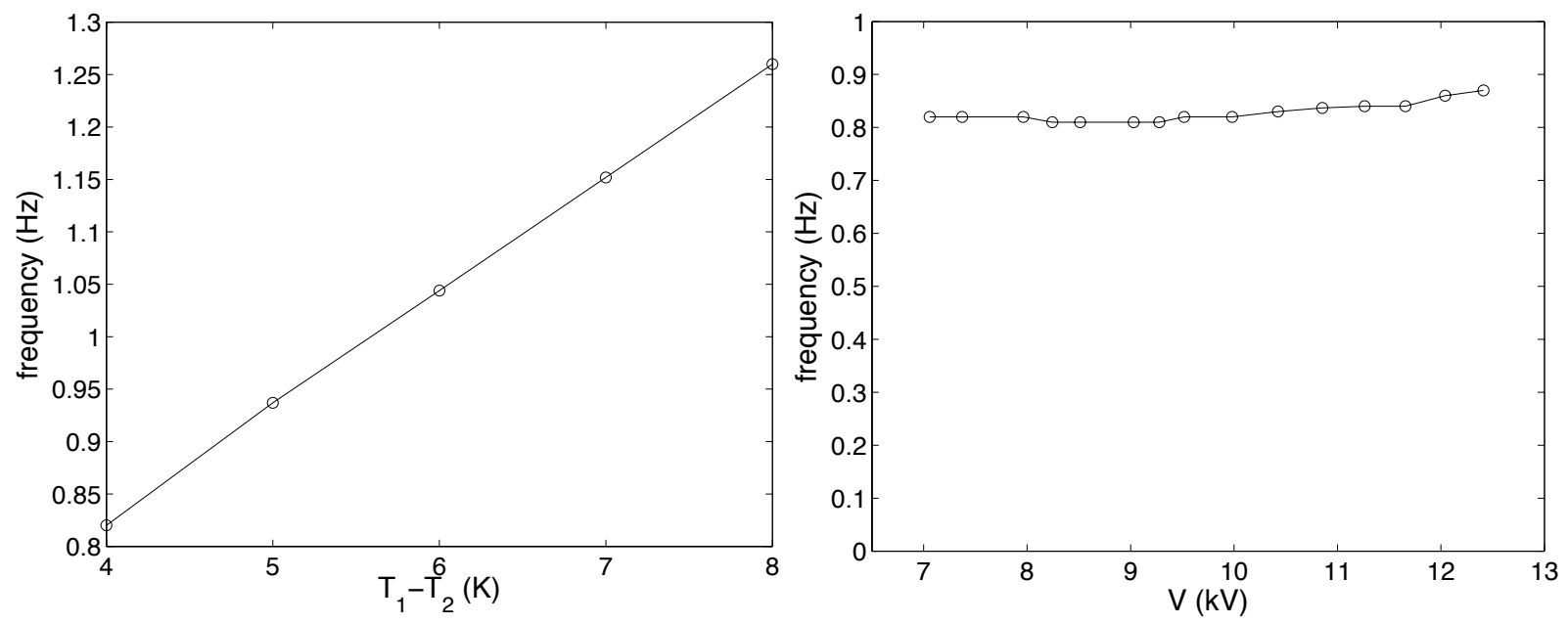

Fig. 5. Dependence of frequency on $\Delta T$ for $V=7.9 \mathrm{kV}$ (left) and on $\Delta V$ for $R a_{g}=4.0 \times 10^{6}(\Delta T=4 \mathrm{~K}$ ) (right) in both cases $\operatorname{Pr}=42.81$, $\eta=0.5$.

Eq. (1). To simulate the $1 / r^{2}$ gravity field, the $1 / r^{5}$ term associated with $R a_{g}$ in Eq. (1) has to be replaced by $1 / r^{2}$.

Beltrame et al. (2003a) have undertaken the linear stability analysis of the pure diffuse state (without convection) computing the critical Rayleigh number $\mathrm{Ra}_{\mathrm{c}}$ of the onset of convection for a fixed aspect ratio. The bifurcated dynamics are explored using the center manifold reduction (Vanderbauwhede and Iooss, 1992) in the parameter space of $R a$ and $\eta$. This center manifold is spanned by the $\ell$ spherical eigenmodes, and leads to a finite dimensional system of ODEs. The problem is intrinsically nonlinear and the ODE system can be computed using the first terms of a Taylor series expansion (Beltrame et al., 2003a), which results in so-called amplitude equations. We do not write these equations because they are already obtained in literature and the precise references are given in the following for each case. However, in order to better understand the role of degeneracy, we recall the general form of these amplitude equations for the more complicated case of this study corresponding to interaction between the odd and even modes, $\ell_{o}$ and $\ell_{e}$, respectively:

$\dot{\mathbf{x}}_{\mathbf{o}}=c_{o} \mathbf{x}_{\mathbf{o}}+c_{o e} \mathbf{P}_{o e}\left(\mathbf{x}_{\mathbf{o}}, \mathbf{x}_{\mathbf{e}}\right)+$ h.o.t.

$\dot{\mathbf{x}}_{\mathbf{e}}=c_{e} \mathbf{x}_{\mathbf{e}}+c_{o o} \mathbf{P}_{\mathbf{o o}}\left(\mathbf{x}_{\mathbf{0}}, \mathbf{x}_{\mathbf{0}}\right)+c_{e e} \mathbf{P}_{\mathbf{e e}}\left(\mathbf{x}_{\mathbf{e}}, \mathbf{x}_{\mathbf{e}}\right)+$ h.o.t.

The odd and even amplitudes, $\mathbf{x}_{\mathbf{o}}$ and $\mathbf{x}_{\mathbf{e}}$, are $2 \ell_{o}+1-$ and $2 \ell_{e}+1$-dimensional vectors, respectively. The polynomials $P_{\text {.. }}$ are $O(3)$-equivariant and are determined using an algebraic algorithm, but the $c$.. coefficients in front of the polynomials are numerically computed and they depend, in particular, on the Prandtl number. First let us remark that some possible quadratic polynomials of the variables $x_{o}, x_{e}$ vanish in the equations. These well-known degeneracies are a consequence of the $O(3)$ symmetry (Chossat et al., 1990).

The self-adjoint degeneracy is a supplementary degeneracy which leads to $c_{e e}=0$ in Eq. (7). This degeneracy comes from the physical anti-symmetry of the two competing force fields, namely $\pm 1 / r^{2}$. The physical anti-symmetry "forces" an anti-symmetry of the pure even modes in the amplitude Eq. (7) since, if the couple $\left(\mathbf{x}_{\mathbf{0}}=\mathbf{0}, \mathbf{x}_{\mathbf{e}}\right)$ is a solution then $\left(\mathbf{x}_{\mathbf{0}}=\mathbf{0},-\mathbf{x}_{\mathbf{e}}\right)$ is a solution too. Recently, it was shown, that this anti-symmetry can be interpreted as a time-reversal symmetry: a transformation of spatial variables and a simultaneous inversion of time $t \rightarrow-t$ (Buono et al., 2006). In the same way as the geometrical symmetries, this physical anti-symmetry leads to a degeneracy in the amplitude equations. In the GEOFLOW framework, we will observe another degeneracy which also leads to anti-symmetry. Even if the following bifurcation analysis will point out the influence of this anti-symmetry, the group-theoretical aspect of this "time-reversal" symmetry is beyond the scope of this paper.

The Taylor series expansion is undertaken at the third order, or if necessary, at the fourth order. Solving the resulting amplitude equations, 3-D time-dependent dynamics can be obtained near the bifurcation point $\left(\mathrm{Ra}^{\mathrm{c}}, \eta\right)$. Furthermore, the analysis is supplemented by the knowledge of the isotropy lattice which gives the different invariant sub-spaces of the phase space and classifies all the possible steadystate by their symmetry (isotropy subgroup.) This method takes place in the equivariant bifurcation theory (Chossat and Lauterbach, 2000).

Near the onset, only one $\ell$ spherical mode is generically unstable. We recall briefly the known results for this codimension 1 case in Sect. 5. More interesting is the case where two consecutive modes $(\ell, \ell+1)$ interact, which is obtained for some critical aspect ratios $\eta_{c}$. This codimension 2 bifurcation is explored in the $(R a, \eta)$ parameters plane in Sect. 6. 


\section{Codimension 1 bifurcation}

In this section, the generic codimension 1 bifurcations for the two central force fields, the $1 / r^{2}$ gravity field and the $1 / r^{5}$ dielectrophoretic field are discussed in turn.

\section{$5.11 / r^{2}$ Gravity field}

If we consider the central self-gravitation field $\left(1 / r^{2}\right)$ only, the non-dimensional buoyancy force of the basic state (without convection) is exactly the opposite of the gravitating force: it is the "self-adjoint" degeneracy. Chossat (1979) gives the consequences on the coefficients of the amplitude equations: the third-order coefficients are negative and the even-order coefficient of the even mode vanish $\left(c_{e e}=0\right.$ of Eq. 7). Then we deduce that the bifurcated branches with maximal isotropy subgroup are supercritical pitchforks.

For the odd mode, the action of the central symmetry alone forces the pitchfork bifurcation. Then, the opposite solutions are in the same $O(3)$-orbit, i.e. they are physically identical solutions applying a $O(3)$ transformation. This is not the case for the even modes, where the two branches of the pitchfork bifurcation correspond to two different physical solutions.

The Fig. 6 presents the $\alpha, \beta$ and $\gamma$ branches, respectively corresponding to the steady states of axial mode- 2 $\left(O(2) \oplus Z_{2}^{c}\right)$, tetrahedral mode-3 $\left(O^{-}\right)$, and cubic mode-4 $\left(O \oplus Z_{2}^{c}\right)$. It is clear that the opposite solution of $\beta$ is obtained by a rotation of $\pi / 2$ around the vertical axis.

\subsection{Dielectrophoretic field}

Let us consider the central dielectrophoretic field $\left(1 / r^{5}\right)$ in a microgravity environment. The "self-adjoint" degeneracy no longer occurs and there is no analytical result. According to the computation of the amplitude equations coefficients (Beltrame et al., 2003a; Beltrame and Egbers, 2004), the bifurcations are supercritical. However, the even branches are slightly different from the self-adjoint case, since the evenorder terms are not negligible. Then, the bifurcation is a perturbed pitchfork one: two asymmetric branches with a hysteresis effect. The details of the bifurcated branches and their stability are presented in Travnikov et al. (2004). The stable steady-states for different aspect ratios $\eta=0.3,0.4$ and 0.5 are presented in Fig. 6.

As already pointed out in Travnikov et al. (2004), the results are similar to the gravity force: the selected branches near the onset are qualitatively equivalent to the gravity force field case. However, for codimension 2 dynamics, the bifurcation is more complex and the influence of the bifurcation type is more important, because heteroclinic cycles appear due to this anti-symmetry of the even pitchfork bifurcation.

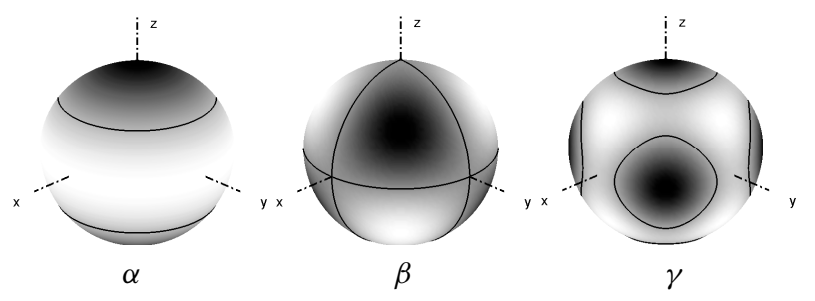

Fig. 6. Radial velocity distribution (dark is the down-welling motion) of the (left to right) $\alpha, \beta$ and $\gamma$ respectively, stable bifurcated branches for $\eta=0.3,0.4$ and 0.5 respectively.

\section{Codimension 2 bifurcation}

The codimension 2 bifurcation occurs for some critical couple $\left(R a_{c}, \eta_{c}\right)$ where two consecutive modes $(\ell, \ell+1)$ interact. In the GEOFLOW framework we found that the $(2,3)$ and the $(3,4)$ mode interaction can be reached for a critical aspect ratio $\eta_{c}=0.33$ and $\eta_{c}=0.45$, respectively (Beltrame et al., 2003a; Beltrame and Egbers, 2004). It is well known that rich dynamics appear in the neighborhood of the bifurcation with complex spatiotemporal structure. One noteworthy type of dynamics are structurally stable heteroclinic cycles (Guckenheimer and Holmes, 1988). Indeed, on one hand, such complex phenomena raise interesting theoretical questions since they are "forced" by the $O(3)$ symmetry group, but also, on the other hand, they occur in different domains of physics. For example, they are reminiscent of the aperiodic Earth's magnetic field. In-depth analysis was undertaken to point out the role of the $O(3)$ group (Chossat and Guyard, 1996), but very little attention has been given to date to the role of the self-adjoint degeneracy. In the following we focus on the $(2,3)$ and $(3,4)$ interactions and explain the underlying dynamics in more detail.

\subsection{Interaction of $(2,3)$ modes}

\subsubsection{Self-adjoint degeneracy}

In this section, we assume that the "self-adjoint" degeneracy occurs in the amplitude equations. Although, it is not the generic case in the GEOFLOW framework, we have shown that for a critical Prandtl number value $P r_{c} \simeq 0.2365$, the same conditions for self-adjoint degeneracy are satisfied (Beltrame et al., 2003a). Considering that the critical $\eta_{c}$ and $\operatorname{Pr}_{c}$ numbers are close to the aspect ratio and Prandtl number of the Earth's outer core, our study is relevant within a geophysical framework.

The numerical results corroborate the theoretical analysis for a self-gravitating case developed in Chossat and Guyard (1996). The cycles connect opposite axisymmetric solutions $\alpha_{ \pm}$of the mode 2, where two different cases occur.

The first, and more simple, case is when the steady-states are on the same axis (Beltrame et al., 2003b). This is the socalled type I heteroclinic cycle. The connections are in the 


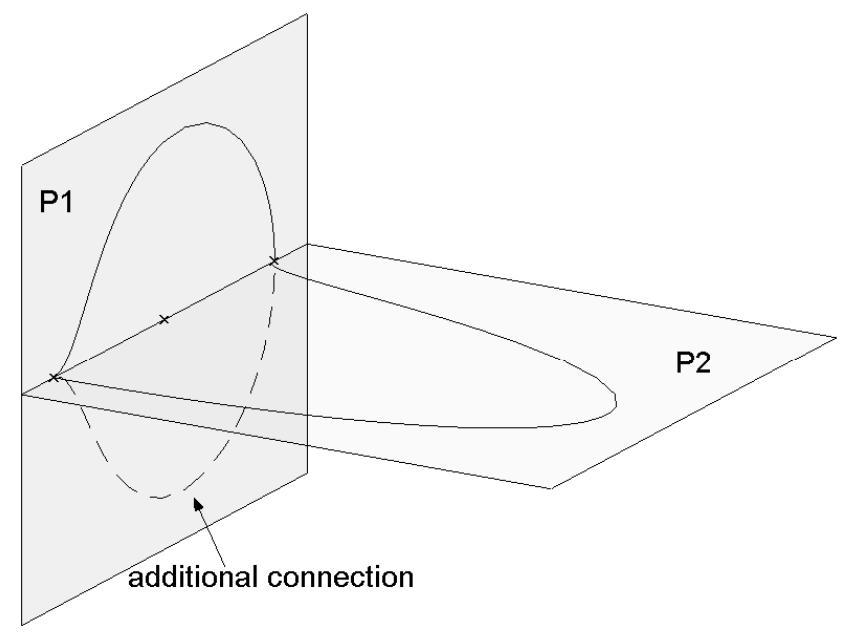

Fig. 7. Additional connection in the Type-I heteroclinic cycle for $R a=1656, \eta=0.3310$ and $P r=0.2365$.

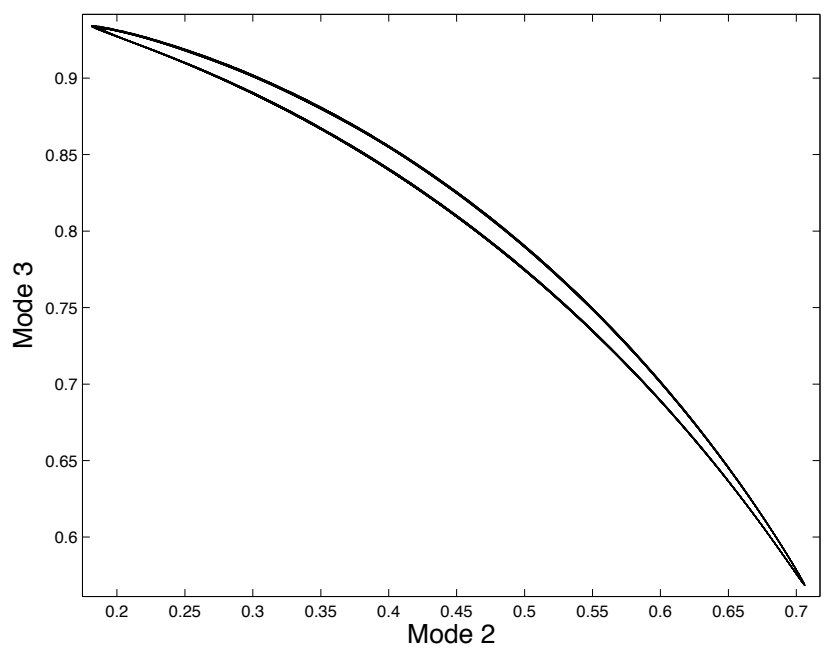

Fig. 8. Trajectories in the energy of the mode 2 and 3 plane for a periodic motion: $R a=1654.5, \eta=0.3308$ and $P r=0.2365$.

invariant planes $P_{1}=$ Fix $\left(\mathrm{O}(2)^{-}\right)$and $P_{2}=$ Fix $\left(\mathrm{D}_{6}^{\mathrm{d}}\right)$, which correspond to the axial symmetry $\left(O(2)^{-}\right)$and to the equilateral triangle symmetry added to the equatorial symmetry $\left(D_{6}^{d}\right)$, respectively. The heteroclinic cycle is stable and attractive, i.e. the dynamics tend to the limit heteroclinic cycle. This last property implies that the dynamics take place near the fixed-point and, due to limitations of numerical accuracy, the trajectory can "jump" to a connection of another cycle. For example, in Fig. 7 the trajectory crosses the $P_{2}$ invariant plane (theoretically impossible) and we observe an additional connection in the plane $P_{1}$. This phenomenon is not only possible in a numerical situation but can also arise in the experiment, since small imperfections can produce such a jump. There is another unstable manifold of both steady states, $\alpha_{ \pm}$, which leads to oscillations in a 4-dimensional in-

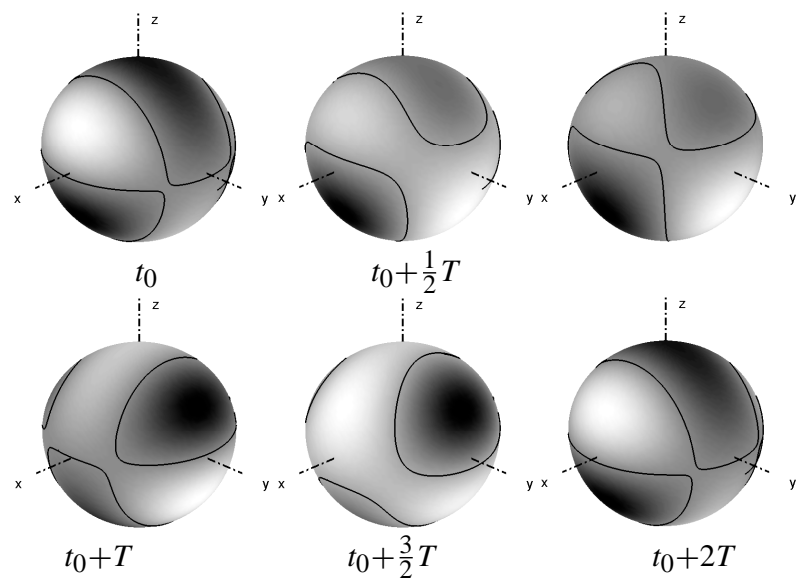

Fig. 9. Radial velocity distribution oscillations during the $2 T$ period for: $R a=1654.5, \eta=0.3308$ and $\operatorname{Pr}=0.2365$.

variant space associated with the $D_{2}^{z}$ symmetry (reflections of two planes). If $T$ designates the period of the energy trajectory loop (Fig. 8), the period of the motion is $2 T$ (two loops). Indeed, the motion at the instant $t+T$ is deduced from the one at the instant $t$ by a geometrical transformation: equatorial reflection followed by a $\frac{\pi}{2}$ rotation of axes $(\mathrm{Oz})$ (Fig. 9).

The second case are cycles called type II heteroclinic cycles, and they involve four axisymmetric solutions with two orthogonal axes (Beltrame and Egbers, 2005). The trajectories, that connect solutions with two different axes, arise in the Fix $\left(\mathrm{D}_{2} \oplus \mathrm{Z}_{2}^{\mathrm{c}}\right)$ invariant plane of mode 2, noted $P_{3}$. The other trajectories (involving the same axis) are in $P_{2}$ or $P_{3}$. When the region of existence of the cycle II coexists with a type I, then the cycle II is broken and the dynamics tends to a type I cycle. Indeed, the expanding and contracting eigenvalues of the connections in $P_{3}$ are very small and of the same order as the vanishing quadratic coefficient (Chossat and $\mathrm{Gu}-$ yard, 1996). Thus, the type I cycle is preferred during numerical simulations. Then, taking $\operatorname{Pr}<P r_{c}$ and $\left|P r-P r_{c}\right| \ll 1$, a cycle II is observed in a very narrow domain of existence in the parameter plane distinct from the cycle I domain. That is most certainly a reason why Chossat and Guyard (1996) were not able to observe it.

Increasing the difference $\left|P r-P r_{c}\right|$, the competition between type-I and II cycles can lead to a complex quasiperiodic motion (Fig. 10). As in the heteroclinic cycles, plateaus close to equilibria appear but they are shorter, and it is difficult to distinguish transitions and equilibria. All steady states involve both modes but we found states, which look like mode 2 axisymmetric $\alpha_{ \pm}$states: see panels (a), (e) and (f) of Fig. 11. The transition (e) to (f) is similar to the one in $P_{2}$, and the transition (d) to (e) is a part of the trajectory in the $P_{1}$ plane. The transition from the state (a) to (d) is due to the expanding value in $P_{3}$, but it does not stay in this plane. It occurs in the 7-dimensional invariant space 


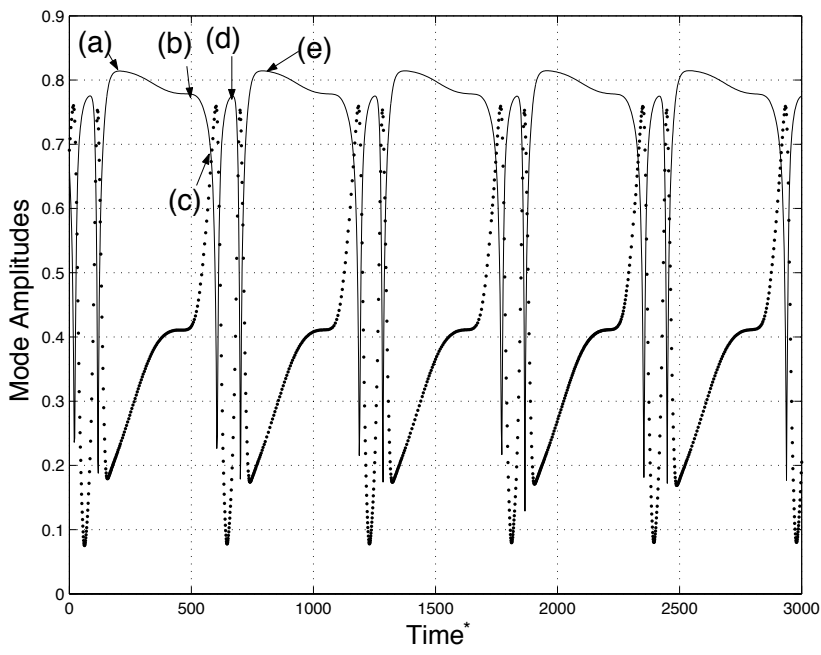

Fig. 10. Evolution of the mode 2 (plain) and mode 3 (dotted) modules during a Generalized Heteroclinic Cycle (GHC) for $R a=1654$, $\eta=0.3306$ and $P r=0.22$.

associated with the $Z_{2}^{-}$symmetry, noted $V$ (one reflection of plane, Fig. 11c). It leads to the $P_{1}$ connection with axial symmetry, but the axis of the axisymmetric steady-states $(a)$ and $4(d)$ differs (Fig. 11). Furthermore, contrary to the type-II cycle, both axes are not orthogonal. The evolution of the axis seems to be random. Indeed, these dynamics are due to the existence of Generalized Heteroclinic Cycles (GHC) found for the $(1,2)$ interaction by Chossat et al. (1999). Because of the $O(3)$-symmetry, there exist multi-dimensional trajectories deduced from those of "simple" heteroclinic cycles and connecting the group orbit of the steady-states. Using the same remarks of Chossat et al. (1999), the existence of the invariant sphere can be proved in our present case (Field, 1986). The simulation shows that actually the trajectories occur on an invariant sphere in $V$, which is 6-dimensional.

\subsubsection{Generic case}

For the silicone oil in the GEOFLOW-experiment, the Prandtl number (see Table 1) is far from the critical value $P r_{c} \simeq 0.2365$. As expected, the coefficient for the quadratic polynomial is not negligible. The coefficients of the amplitude equations do not vary much between the different fluids and, as a result, the bifurcated dynamics do not present important differences. Both axisymmetric solutions $\alpha_{ \pm}$with different sense of convection still exist but there is a factor 10 between their amplitudes. The greater amplitude solution, here $\alpha_{-}$, is stable versus the mode 2. Furthermore the connection in $P_{3}$ from $\alpha_{+}$to a copy of $\alpha_{-}$still exists. However, the back connection in $P_{3}$ is broken and the trajectory tends to a mixed-modes equilibrium. Thus, there is no longer a heteroclinic cycle. The selected bifurcated branch depends on the $\alpha_{-}$stability versus the isotypic components of the mode 3 . Either $\alpha_{-}$is stable or $\beta$ of the mode 3 is stable. This com-

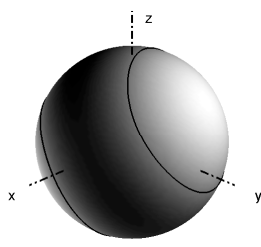

(a)

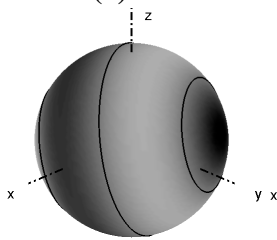

(d)

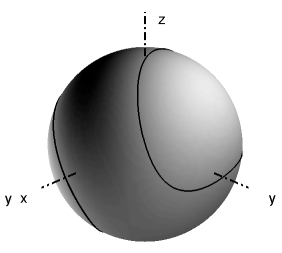

(b)

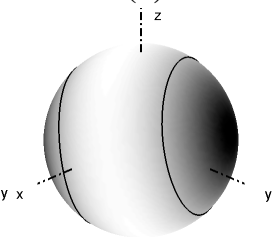

(e)

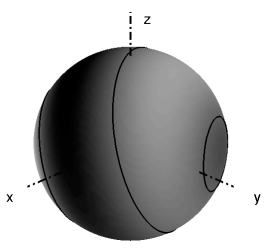

(c)

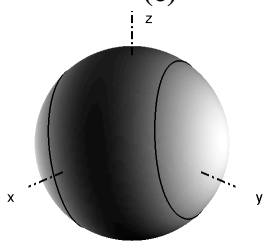

(f)
Fig. 11. Radial velocity distribution for the different states of the Fig. 10 during the GHC.

petition does not lead to time-dependent dynamics and we observe only steady-states.

\subsection{Interaction of the $(3,4)$ modes}

For the $(3,4)$ interaction, the situation is quite different because we have proved that the heteroclinic cycle does not exist for the self-adjoint case (Beltrame, 2006b). This is a consequence of the $O(3)$ symmetry. More precisely, invariant planes break the possible connections between steady states.

However, we have shown that another degeneracy can occur for the experimental fluid (Beltrame and Egbers, 2004): $c_{o o}=0$ in the even amplitude Eq. (7). The consequence is the existence of pure mode 3 branches which are generically mixed with the mode 4 (Beltrame, 2006b). In this way, the degeneracy induces degenerated isotropy subgroups. In these subgroups the dynamics have an anti-symmetry property: the solution is exactly the opposite by a geometrical transformation. The simulation of the amplitude equations developed up to third order shows complex and stable heteroclinic cycles (Beltrame, 2006a $\mathrm{a}^{1}$ ). We can distinguish a heteroclinic cycle and homoclinic cycle. The first one involves the cubic solution of the mode $4(\gamma)$ and the tetrahedral solution of the mode- $3(\beta)$ and the second one connects the solutions of the orbit of $\beta$. According to the simulation these cycles alternate indefinitely but not periodically (Beltrame, $\left.2006 a^{1}\right)$. Nevertherless, the direct simulation with the numerical code used in the first part only lets the homoclinic cycle appear. The dynamics after some cycles tend to the $\gamma$ solution which seems to be stable (Fig. 12). Indeed, the amplitude of $\gamma$, due to the hysteresis, is not very small at the onset of bifurcation $(\simeq 0.3)$. Then, the fourth order terms can have a non-negligible influence on the bifurcation diagram. The computation at the fourth order in the amplitude equations shows that the $\gamma$ solution is actually stable in the supercritical region. Hence the heteroclinic cycle previously mentioned cannot occur. 


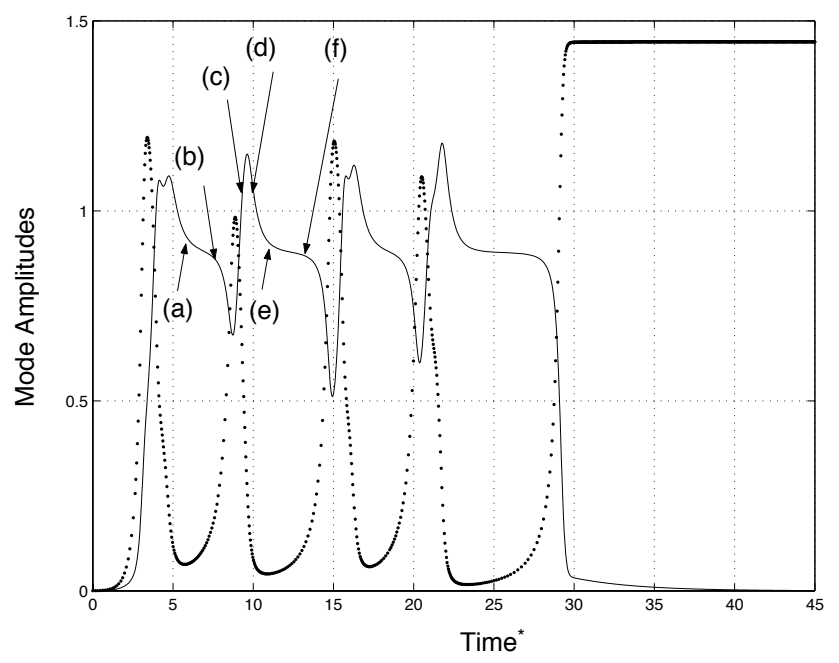

Fig. 12. Evolution of the mode 3 (plain) and mode 4 (dotted) amplitudes during a homoclinic cycle for $R a=2205, \eta=0.45$ and $\operatorname{Pr}=107.22$.

However, this homoclinic cycle remains interesting because the symmetry is completely broken during the transition (Fig. 13c ). The time spent near the $\beta$ equilibrium is short and the dynamics seem to slide onto the $\beta$-orbit showing a slight rotation (Figs. 13a and b). The axis of the different $\beta$ equilibria connected in the cycle seem to be random. Nevertheless, this behavior is different to the GHC of the $(2,3)$ interaction because it does not derive from a simple cycle. Furthermore, an invariant sphere does not exist, which is an important key of the GHC.

The amplitude of the mode 4 for the last equilibrium before the final transition to $\gamma$ is smaller than for the other equilibria of the cycle (Fig. 12). Indeed, there exist two equilibria, $\beta$ and $\beta^{\prime}$, very near to each other, which are almost pure mode 3 with the $O^{-}$tetrahedral symmetry. Taking the small amplitude of the mode 4 into account, the analytical resolution of the amplitude equations show that $\beta$ is actually in the mixed-modes $\operatorname{Fix}\left(\mathrm{O}^{-}\right)$plane and $\beta^{\prime}$ is in the 3-dimensional space $\operatorname{Fix}\left(\mathrm{D}_{4}^{\mathrm{d}}\right)$. This last invariant space contains $\operatorname{Fix}\left(\mathrm{O}^{-}\right)$, hence $\beta^{\prime}$ has less symmetry than $\beta$. According to the numerical results, the homoclinic cycle involves the $\beta^{\prime}$ orbit, while the heteroclinic cycle in Beltrame $(2006 a)^{1}$ involves $\beta$. When the $\beta$ equilibrium is present in the homoclinic cycle, then the same transition as in Beltrame (2006a) ${ }^{1}$ leads to the stable $\gamma$ equilibria and ends the cycle. Although the homoclinic connections disappear after some cycles, the duration of this transition is large enough to be observable during the experiment. Furthermore, they persist far away from the onset of convection, contrary to the other studied cycles.

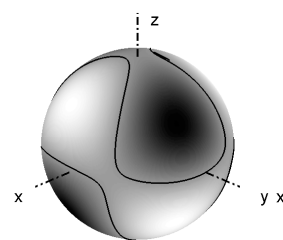

(a)

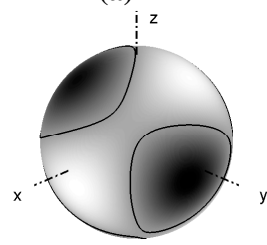

(d) (b)

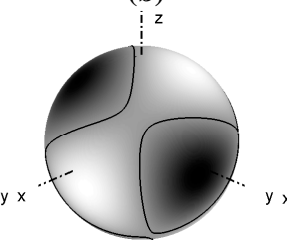

(e)

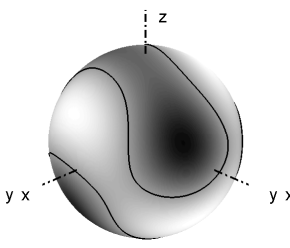

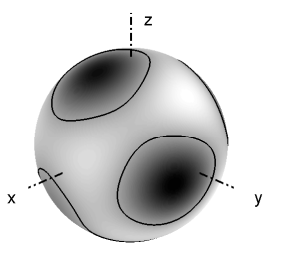

(c)

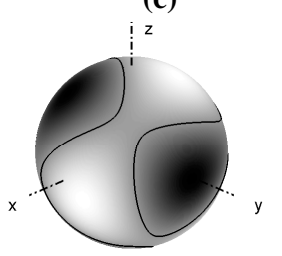

(f)
Fig. 13. Radial velocity distribution for the different states of the Fig. 12 during a homoclinic cycle.

\section{Conclusions}

The specific features of the central force field due to the dielectrophoretic effect is studied using a pseudospectral numerical code and a bifurcation analysis near the onset of convection. In terrestrial conditions as well as in a microgravity environment, the codimension 1 bifurcation which presents only steady-states or rotating waves, is only slightly modified from the case with another force field. On the contrary, the complex time-dependent dynamics show a significant difference. For the terrestrial conditions with a dielectrophoretic field, oscillations appear for relative small Rayleigh numbers compared to the case without dielectrophoretic force. In the microgravity environment within the dielectrophoretic field, the dynamics depend strongly on the Prandtl number in contrast to the self-gravitating force field. For a critical Prandtl number $\left(P r_{c} \simeq 0.24\right)$, the simulation of the GEOFLOW experiment presented a rich variety of heteroclinic cycles of the $(2,3)$ mode interactions, which can also occur in the astrophysical framework. Unfortunately, for the experiment such low Prandtl number values cannot be reached. However, we have observed a complex homoclinic cycle, which satisfies the requirements on the Prandtl number. These dynamics do not arise within the astrophysical framework. Thus the dielectrophoretic force leads to a rich variety of timedependent dynamics, which are not always present for gravity forces. Finally, these simulations point out the limit of the geometrical symmetries as bifurcation mechanisms, since the presence of the dielectrophoretic force field does not break the symmetry of the other case. The anti-symmetry plays a relevant role for the existence of the heteroclinic cycles. It would be interesting to take into account this invariant to better understand the mechanism of such dynamics.

Beyond these theoretical aspects, there are many outlooks of geophysical interest. Firstly, the study of $r$-dependent gravity fields is relevant for the Earth's mantle. Recently, Früh (2005) has compared different central force fields of 
the form $g=C r^{n}$ where $n=5$ to $n=-5$ for an axisymmetric case. Früh has shown that the convection rolls are qualitatively similar for all the values of $n$ according to our result of the Sect. 5. But as we have undertaken for the $1 / r^{2}$ and $1 / r^{5}$-dependent force fields, it would be interesting to look at possible 3-D-time-dependent dynamics under an $r$ dependent force field.

Secondly, although the non-rotating case leads to very rich kind of dynamics, the rotating case is, of course, relevant future work which has to be exploited for the GEOFLOWexperiment as underlined by the results in Gellert et al. (2005).

Edited by: W.-G. Früh

Reviewed by: three referees

\section{References}

Amara, K. and Hegseth, J: Convection in a spherical capacitor; J. Fluid Mech., 450, 297-316, 2001.

Beltrame, P.: Heteroclinic cycles for the $(3,4)$ mode interaction in astrophysical framework, in print, $2006 \mathrm{~b}$.

Beltrame, P. and Egbers, C.: The $(3,4)$ Mode interaction in the GeoFlow Framework, Proc. Appl. Math. Mechanics, 4, 474-475, 2004.

Beltrame, P. and Egbers, C.: Heteroclinic Cycles of Type II in the $(2,3)$ Interaction in the GEOFLOW-Experiment, edited by: Peinke, J., Kittel, A., Barth, S., and Oberlack, M., Progress in Turbulence, 101, 133-136, 2005.

Beltrame, P., Egbers, C., and Hollerbach, R.: The Geoflowexperiment on ISS (part III): Bifurcation analysis, J. Adv. Space Res., 32, 191-197, 2003a.

Beltrame, P., Egbers, C., and Travnikov, V.: Heteroclinic Cycles in the Geoflow-experiment on the International Space Station, edited by: Marquès, F. and Meseguer, A., Nonlin. Dyn. Fluids, 171-174, 2003b.

Buono, P. L., Lamb, J. S. W., and Roberts, M.: Bifurcation and branching of equilibria in reversible equivariant vector fields, in print, 2006.

Canuto, C., Hussaini, M. Y., Quarteroni, A., and Zang, T.: Spectral Methods in Fluid Dynamics, Springer, Berlin, 1987.

Chossat, P.: Bifurcation and stability of convective flows in a rotating or nonrotating spherical shell, SIAM J. of Appl. Math., 37, 624-647, 1979.

Chossat, P., Lauterbach, R., and Melbourne, I.: Steady-State Bifurcation with $O(3)$-Symmetry, Arch. Rational Mech. Anal., 113, 313-376, 1990.

Chossat, P. and Guyard, F.: Heteroclinic cycles in bifurcation problems with $O(3)$ symmetry and in the Spherical Bénard Problem, J. Nonlin. Sci., 6, 201-238, 1996.
Chossat, P., Guyard, F., and Lauterbach, R.: Generalized Heteroclinic Cycles in Spherically Invariant Systems and Their Perturbations, J. Nonlin. Sci., 9, 479-524, 1999.

Chossat, P. and Lauterbach, R.: Methods in Equivariant Bifurcations and Dynamical Systems, World Scientific, Singapore, 2000.

Egbers, C., Beyer, W., Bonhage, A., Hollerbach, R., and Beltrame, P.: The GEOFLOW - experiment on ISS (Part I): Experimental preparation and design, Adv. Space Res., 32/2, 171-180, 2003.

Field, M.: Equivariant bifurcation and symmetry breaking, J. Dyn. Diff. Eq., 1, 369-421, 1986.

Friedrich, R. and Haken, H.: Static, wavelike, and chaotic thermal convection in spherical geometries, The American Physical Society, 34(3), 2100-2120, 1986.

Früh, W.-G.: Using magnetic fluids to simulate convection in a central force field in the laboratory, Nonlin. Processes Geophys., 12, 877-889, 2005, http://www.nonlin-processes-geophys.net/12/877/2005/.

Futterer, B., Hollerbach, R., and Egbers, C.: Periodical convective flow in spherical shells, Proc. Appl. Math. Mech., 4(1), 482-483, 2004.

Gellert, M., Beltrame, P., Travnikov, V., and Egbers, C.: The GEOFLOW experiment - spherical Rayleigh-Bénard convection under the influence of an artificial force, J. of Physics, Volume Conf. Series, 14, 157-161, 2005.

Guckenheimer, J. and Holmes, P.: Structurally stable heteroclinic cycles, Math. Proc. Camb. Phil. Soc., 103, 189-192, 1988.

Hart, J. E., Glatzmaier, G. A., and Toomre, J.: Space-laboratory and numerical simulations of thermal convection in a rotating hemispherical shell with radial gravity, J. Fluid Mech., 173, 519544, 1986.

Hollerbach, R.: A spectral solution of the magneto-convection equations in spherical geometry, Int. J. Num. Meth. Fluids, 32, 773-797, 2000.

Pohl, H. A.: Dielectrophoresis, Cambridge University Press, 1978.

Porter, J. and Knobloch, E.: New type of complex dynamics in the 1:2 spatial resonance, Physica D, 159, 125-154, 2001.

Sitte, B.: Thermische Konvektion in Zentralkraftfeldern, Ph.-D. Thesis, ZARM, University of Bremen, 2003.

Travnikov, V., Egbers, C., and Hollerbach, R.: The GEOFLOWExperiment on ISS (Part II): Numerical simulation, Adv. Space Res., 32, 181-189, 2003.

Travnikov, V., Beltrame, P., and Egbers, C.: The $(3,4)$ Mode Interaction in the GeoFlow Framework, in MHD Couette Flows, American Institute of Physics, 4, 45-53, 2004.

Travnikov, V.: Thermische Konvektion im Kugelspalt unter radialem Kraftfefld, Ph.-D. Thesis, Cuvillier, Goettingen (in German), 2004.

Vanderbauwhede, A. and Iooss, G.: Center manifold theory in infinite dimensions. Dynamics Reported, 1 new series , 123-163, 1992.

Yavorskaya, I., Fomina, M., Balyaev, N. I,. and Yu., N.: A simulation of central-symmetry convection in microgravity conditions, Acta Astronautica, 11, 179-183, 1984. 